\title{
REAL-TIME POLLUTION ANALYSIS AND LOCATION BASED ON VEHICLE PARTICLE RADAR Jing Gao ${ }^{1}$, Xu Wang ${ }^{1}$,Yuefeng Zhao ${ }^{{ }^{*}}$,Junyong Zhao ${ }^{1}$ \\ ${ }^{1}$ School of Physics and Electronic Science, Shandong Normal University, 250014, Jinan *Email:yuefengzhao@126.com
}

\begin{abstract}
This paper first studies the advantages and main observation methods of atmospheric particulate matter lidar in Regional large-scale measurement, and then discusses the measurement principle of lidar and two commonly used methods for inversion of aerosol extinction coefficient.Finally, the mobile observation system consisting of lidar-assisted vehicle particle monitor is used. Anping County is used as the research area.The lidar data of Anping County on September 15 to September 20, 2017 are obtained by means of the combination of navigation and fixed vertical monitoring. The results of fixed detection showes that foreign pollutants begin to be imported around $10 \mathrm{pm}$ on the $15 \mathrm{th}$, and the superimposed pollutants peakes around $2 \mathrm{pm}$ on the 16th. The concentration of particulate matter gradually decreased after $2 \mathrm{pm}$ on the 16th, which is in line with the trend of air quality rising first and then falling in accordance with the trend of the Ministry of Environmental Protection.According to the particle trajectory tracking map of the navigation area, the contaminant transport was analyzed Combined with the meteorological conditions at that time.
\end{abstract}

\section{INTRODUCTION}

Suspended particulate matter or aerosols in the atmosphere, especially fine particles, lead to reduced visibility, frequent haze and other atmospheric pollution and cause damage to human health, making the monitoring, remediation and prevention of particulate matter a hot $\operatorname{spot}^{[1]}$.For the measurement method of the particulate matter, some physical and chemical properties such as mass and concentration can be obtained from the ground observation, but the ground measurement instrument cannot obtain the spatial distribution information of the particulate matter ${ }^{[2]}$. Although the vertical distribution of particulate matter can be grasped by means of air balloon detection, airborne detection orsatellite remote sensing, it is difficult to realize the continuity of the data because of the high operation cost.Atmospheric particulate matter lidar is mainly used for atmospheric transmission process monitoring and Regional large-scale measurement ${ }^{[3]}$.The auxiliary vehicle particulate matter monitor realizes rapid movement monitoring of emission sources, and monitors the main emission sources that affect atmospheric conditions in real time, providing effective data support for pollution source analysis and related decision-making.

Common monitoring methods include: plane scanning, mobile navigation and fixed vertical monitoring.

\section{METHODOLOGY}

Lidar equation $^{[4]}$ :

$P_{L}\left(z, \lambda_{L}\right)=K_{L} \frac{1}{z^{2}} \beta\left(z, \lambda_{L}\right) \exp \left\{-2 \int_{z_{0}}^{z} \alpha\left(z^{\prime}, \lambda_{L}\right) d z^{\prime}\right\}(1)$

Here, $P_{L}\left(z, \lambda_{L}\right)$ is the atmospheric backscattered echo power (W) at the height $\mathrm{z}$ received by the lidar; $\lambda_{L}$ is the laser wavelength $(\mathrm{nm}) ; K_{L}$ is the lidar system constant $\left(W \cdot \mathrm{km}^{3} \cdot \mathrm{sr}^{-1}\right) ; \beta\left(z, \lambda_{L}\right)$ is the backscattering coefficient of the atmosphere at the height $\mathrm{z}, \beta\left(z, \lambda_{L}\right)=\beta_{m}\left(z, \lambda_{L}\right)+\beta_{a}\left(z, \lambda_{L}\right)$, $\beta_{m}\left(z, \lambda_{L}\right)$ and $\beta_{a}\left(z, \lambda_{L}\right)$ are the backscattering coefficients of air molecules and atmospheric aerosols respectively; $\alpha\left(z, \lambda_{L}\right)$ is the extinction coefficient $\left(\mathrm{km}^{-1}\right)$ of the atmosphere at the height $\mathrm{z}, \alpha\left(z, \lambda_{L}\right)=\alpha_{m}\left(z, \lambda_{L}\right)+\alpha_{a}\left(z, \lambda_{L}\right)$,

$\alpha_{m}\left(z, \lambda_{L}\right)$ and $\alpha_{a}\left(z, \lambda_{L}\right)$ are the extinction coefficient of air molecules and atmospheric aerosols respectively; ${ }^{z_{0}}$ is the height where the lidar is located.

There are two main methods for solving the Mie- scattering lidar equation: the Klett method and the Fernald method ${ }^{[5-6]}$.

Klett method: klett assumes that the atmospheric extinction coefficient and backscattering coefficient satisfy: 
$\beta\left(z, \lambda_{L}\right)=\frac{\alpha\left(z, \lambda_{L}\right)^{k}}{S_{1}}$,

Here, $\mathrm{k}$ is a constant, the range of variation is 0.7 to 1.3 , and the typical value is $1 ; \mathrm{S}_{1}$ is a constant.

Substituting equation (2) into equation (1), the atmospheric backscatter coefficient is eliminated, and a Bornoulli equation is obtained. Solve this equation and get the atmospheric extinction coefficient:

$\frac{\alpha\left(z, \lambda_{L}\right)=}{\exp \left\{\left[S\left(z, \lambda_{L}\right)-S\left(z_{c}, \lambda_{L}\right)\right] / k\right\}}$

Here, $S\left(z, \lambda_{L}\right)=\ln \left[P_{L}\left(z, \lambda_{L}\right) z^{2}\right] ; z_{c}$ is the height at which the boundary value is selected. The Klett method is suitable for areas with high concentrations of atmospheric aerosols, such as atmospheric boundary layers, clouds, and aerosol layers with large optical thicknesses.

Fernald method: Fernald assumes the following relationship:

$\alpha_{a}\left(z, \lambda_{L}\right)=S_{1} \beta_{a}\left(z, \lambda_{L}\right)$

$\alpha_{m}\left(z, \lambda_{L}\right)=S_{1} \beta_{m}\left(z, \lambda_{L}\right)$

$\mathrm{S}_{1}$ is the extinction backscattering ratio of atmospheric aerosols, also known as the lidar ratio. $S_{2}$ is the extinction backscattering ratio of air molecules, which can be derived from Rayleigh scattering theory.

Substituting equations (4) and (5) into equation (1) eliminates the extinction coefficient of atmospheric aerosols, and simplifies to obtain a Bernoulli equation. Solving this equation yields the backscattering coefficient of atmospheric aerosols:

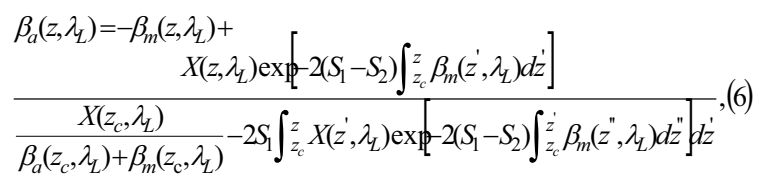

Using the atmospheric aerosol backscattering coefficient obtained from the inversion and the assumed lidar ratio, the extinction coefficient of the atmospheric aerosol can be calculated according to formula (6).

\section{Field monitoring}

The purpose is to use the lidar navigation and fixed monitoring to analyze the trend of pollutants over the city and the causes of urban Pollution by

combining the meteorological conditions .

\subsection{Urban air quality analysis}

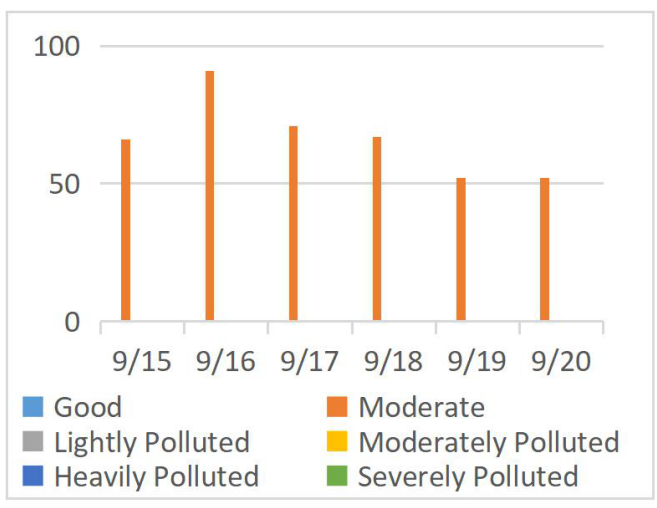

Figure 1 Anping County Air Quality Daily

Fig.1 shows that the air quality in Anping County is good in recent days, but the pollution index on September 16 is significantly higher than the pollution index in other days, and it shows a trend of rising first and then falling.

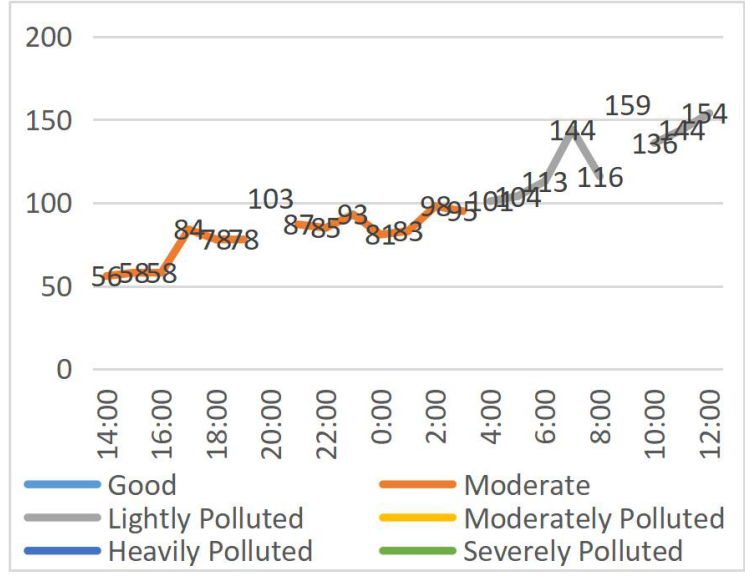

Figure 2 Air quality trend of Anping County in the past 24 hours

It can be seen from Fig.2that the air quality index began to rise at 2 am on September 16 and reached its maximum at around 9 am, and then floats up and down. This paper aims to combine the causes of this phenomenon with meteorological and environmental analysis based on radar detection data.

\section{2 system parameters}

The laser uses a pumped solid-state laser with a working wavelength of $532 \mathrm{~nm}$, a single-pulse output energy of $\geq 1 \mathrm{~mJ}$, and a pulse frequency of $2 \mathrm{kHz}$. The telescope has a diameter of 
$180 \mathrm{~mm}$. The particle monitor has a measurement range of $0-100 \mathrm{mg} / \mathrm{m} 3$ and an accuracy of less than $\pm 10 \%$. The wind speed and direction meter has two measurement range modes of 0 to $70 \mathrm{~m} / \mathrm{s}$ and 0 to 360 degrees with an accuracy of $\pm 3^{\circ}$.

\section{Analysis of the overall urban pollution}

\subsection{Fixed monitoring}
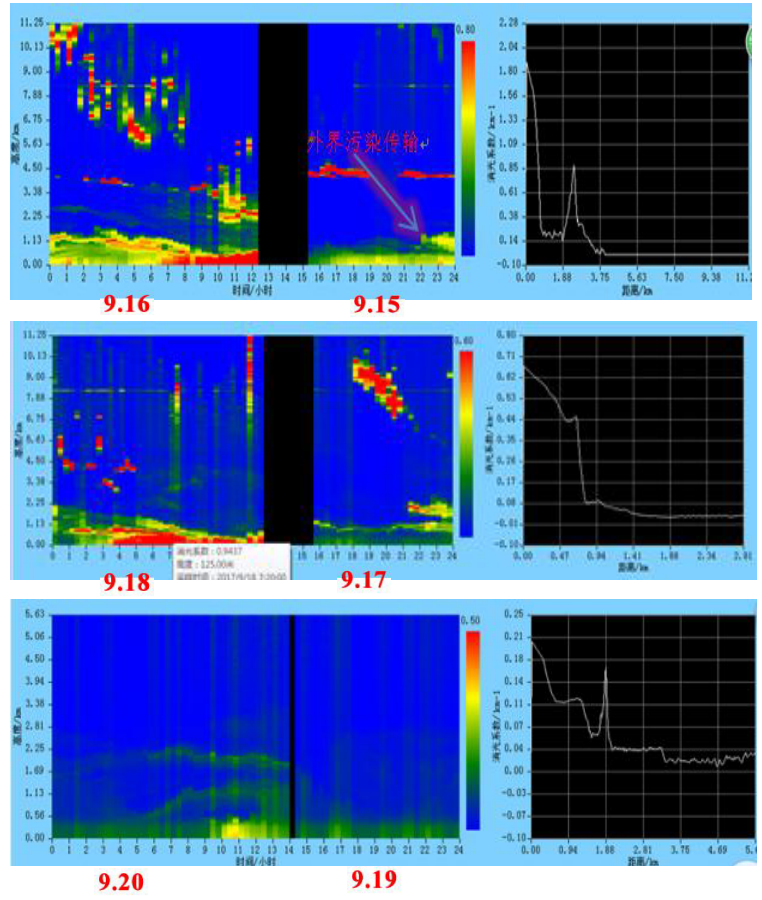

Figure 3Temporal and spatial evolution map of fixed particle concentration on September 15 to September 20, 2017

articulate matter concentration monitored from September 15 to September 20, 2017

As shown in Fig.3, the concentration of particulate matter was the highest on the 16th, and mainly the foreign input pollution. On the 19 th, the concentration of particulate matter was the lowest and the air quality was the best. At around $10: 00$ on the 15 th, foreign pollutants began to be transported.By about $2 \mathrm{pm}$ on the 16 th, the external pollutants and local pollutants were superimposed to reach a peak, and then the concentration of particulate matter gradually decreased.At around 21:00 on the 17th, there was another pollution and it was superimposed with local pollution to reach the peak at around 6:00 on the 18th. The particulate matter concentration then decreased and dropped to a minimum on the 19th. On the 19th and 20th, the concentration of particulate matter was lower and the air quality was better. On the 20th, around 10:00, there was a temporary increase in the concentration of particulate matter, which was caused by dust.

\subsection{Mobile navigation monitoring}

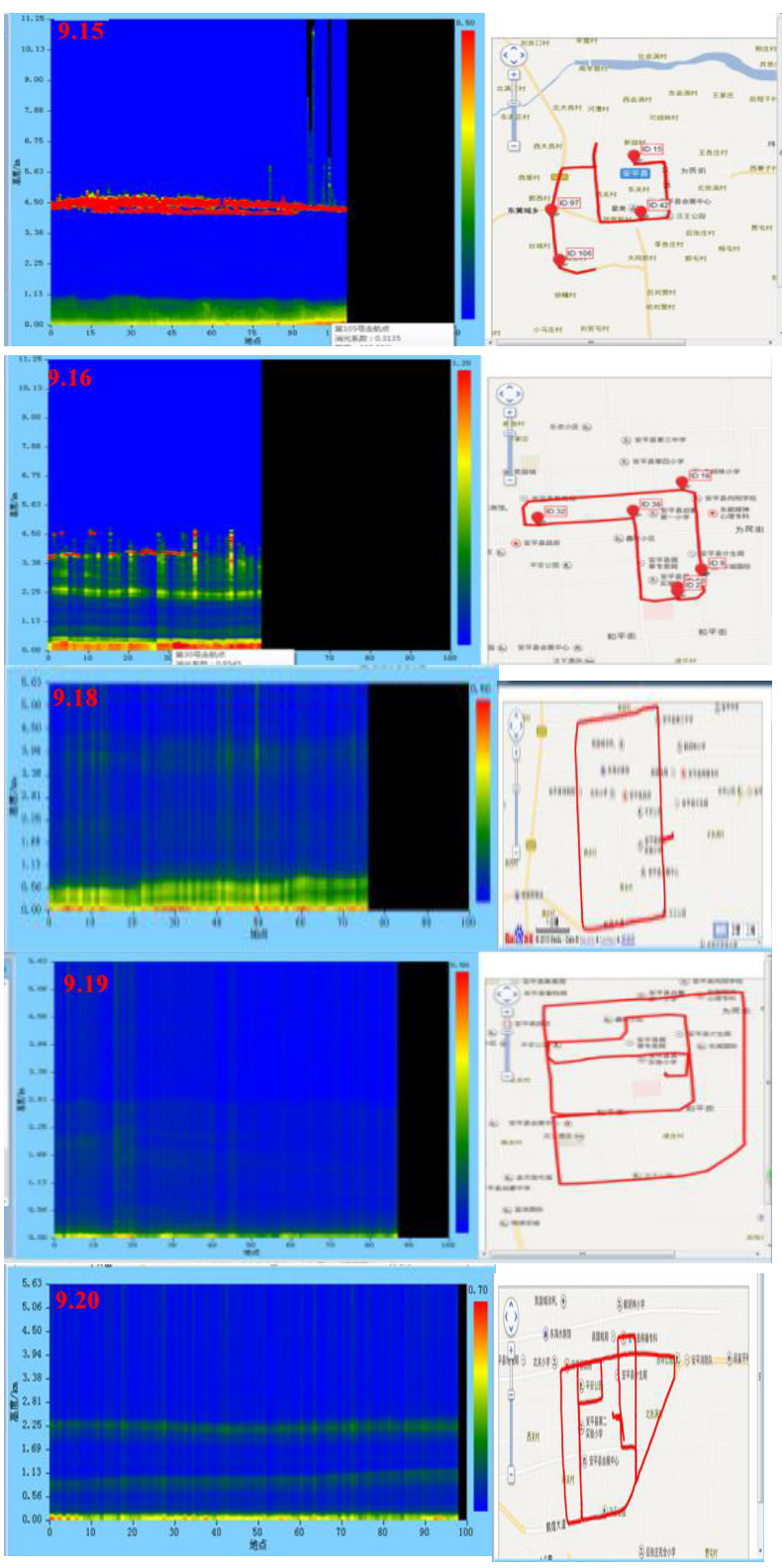

Figure4 Spatio-temporal evolution of particulate matter concentration in the navigation on September 15 to September 20, 2017

As shown in Fig.4, the concentration of particulate matter in Anping County was the lowest on the 19th and the highest on the 16th. The total inversion results show that from the 15 th, the main pollution is some pollution near the surface, and the clouds in the air are thicker, which can be seen as cloudy days. According to the detection results, atmospheric particulate matter pollution began to aggravate on the 16th. At the beginning of the 18th, atmospheric 
particulate matter pollution was significantly weakened, and the inversion results from 19th to $20^{\text {th }}$ remained basically unchanged.Compared with the urban air quality analysis report of Anping County from September 15 to September 20, the inversion results are consistent with the air quality forecast provided by the Ministry of Environmental Protection.

\section{3analysis}

By voyage, it can be found that the concentration of particulate matter in Hehuang Avenue is higher than that in other sections, and the concentration of particulate matter is significantly reduced from Hehuang Avenue to Hanwang Park. It can be inferred that the pollution source on Hehuang Avenue is mainly the discharge of motor vehicle exhaust and the dust generated by the motor vehicle when driving. The concentration of particulate matter in Yuhua Road and Hongqi Street is also high. Due to the laying of gas pipelines on Yuhua Avenue and Hongqi Street, the laying of gas pipelines causes dust, resulting in high concentration of particulate matter in this section. Finally, the EPA's particle concentration is also too large, there are construction sites around it, causing the spread of dust, and because of the surrounding buildings around the EPA, the diffused pollutants cannot be dispersed in time.

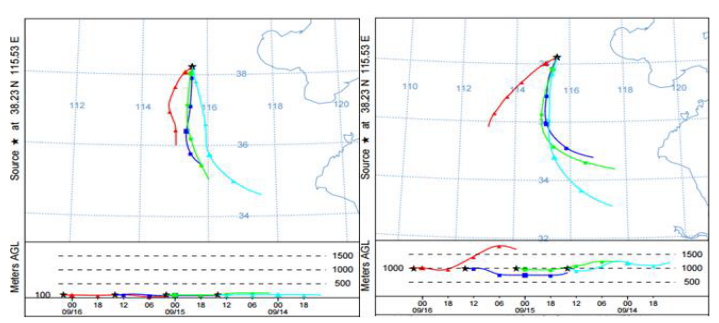

Figure 5 particle backward trajectory tracking

It can be seen from Fig. 5 that the wind direction of the navigation area is the southeast wind. The low-altitude diffusion is mainly at 100 meters, and the low-level diffusion is mainly the exhaust of motor vehicles. At 1000 meters, the main source of pollutants is high-altitude diffusion, while the high-level diffusion pollutants are mainly from the transmission of external pollution.At the same time, there are fewer vehicles on Hanwang Road, Hongqi Street and Beixin Avenue, and the concentration of particulate matter is relatively low. It can be speculated that the pollution source near the provincial road 301 is mainly the exhaust of the motor vehicle exhaust.

\section{RESULTS}

Based on the on-board particulate matter lidar mobile observation system, this paper monitors the weather conditions of Anping County from September 15 to September 20, 2017, and provides a comparison with the Ministry of Environmental Protection,confirming the accuracy and practicability of the system.

Vertical monitoring can directly reflect changes in urban pollution, while navigation monitoring can intuitively reflect regional pollution. The navigation detection adds the maneuverability of the detection based on the fixed detection, which is beneficial to better realize the rapid movement monitoring and real-time monitoring of the emission source, and provide effective data support for pollution source analysis and related decision-making.

\section{ACKNOWLEDGEMENTS}

The research work was supported by National $\mathrm{N}$ atural Science foundation of China under Grant No.81400285. And the authors would also like $t$ o gratefully acknowledge the support from the $\mathrm{k}$ ey research and development foundation of Sha ndong Province under Grant No.2016GGX1010 16.

\section{REFERENCES}

[1]Inaba H.,Opto-electronics,4(2),101-123(1972).

[2]Reinhardt, A., In 2016 46th European Microwave Conference (EuMC) (pp. 1509-1512). IEEE.

[3]Tao, J.,Science China Earth Sciences, 56(8), 1422-1433(2013).

[4]F. G. Fernald, Appl. Opt. 23, 652-653 (1984).

[5]Mao F.,et al.Optics express,21(7), 8286-8297(2013)

[6]Ji H, et al. Journal of Quantitative Spectroscopy and Radiative Transfer 188 (2017): 71-78. 